\title{
Testing the Stability of the Fine Structure Constant in the Laboratory
}

\author{
N. Kolachevsky • A. Matveev • J. Alnis • C.G. Parthey • \\ T. Steinmetz • T. Wilken • R. Holzwarth · T. Udem • \\ T.W. Hänsch
}

Received: 10 April 2009 / Accepted: 8 June 2009 / Published online: 5 August 2009

(C) The Author(s) 2009. This article is published with open access at Springerlink.com

\begin{abstract}
In this review we discuss the progress of the past decade in testing for a possible temporal variation of the fine structure constant $\alpha$. Advances in atomic sample preparation, laser spectroscopy and optical frequency measurements led to rapid reduction of measurement uncertainties. Eventually laboratory tests became the most sensitive tool to detect a possible variation of $\alpha$ at the present epoch. We explain the methods and technologies that helped to make this possible.
\end{abstract}

Keywords Drift of the fine structure constant · Frequency comb · Laser stabilization

PACS 06.20.Jr $\cdot 06.30 . \mathrm{Ft} \cdot 32.30 . \mathrm{Jc}$

\section{Introduction}

The fine structure constant $\alpha=e^{2} / \hbar c$ is a dimensionless parameter that measures the strength of all electromagnetic interactions. As such it appears in a large variety of phenomena such as forces between charged objects that in turn determine the structure of atoms and molecules. Further examples are the propagation of electromagnetic waves, chemical reactions or even macroscopic phenomena like friction. The value of the fine structure constant can be thought of as the electromagnetic force between two electrons at a distance of

N. Kolachevsky · A. Matveev · J. Alnis · C.G. Parthey · T. Steinmetz · T. Wilken · T. Udem • T.W. Hänsch

Max-Planck Institut für Quantenoptik, Hans-Kopfermann-Str 1, 85748 Garching, Germany

N. Kolachevsky $(\varangle) \cdot$ A. Matveev

P.N. Lebedev Physical Institute, Leninsky prosp. 53, 119991 Moscow, Russia

e-mail:kolik@lebedev.ru

T. Steinmetz $\cdot$ R. Holzwarth

MenloSystems GmbH, Am Klopferspitz 19, 82152 Martinsried, Germany

T.W. Hänsch

Ludwig-Maximilians-Universität, Munich, Germany 
one meter measured in units where the speed of light $c$ and Planck's constant $\hbar$ are set to unity. The fine structure constant is used as an expansion parameter in the quantum theory of electromagnetic interactions, Quantum Electrodynamics (QED) which is one of the most successful theories in physics.

Unfortunately, neither this theory nor any other known theory makes any prediction on the value of the fine structure constant which is determined experimentally to $\alpha \approx 1 / 137$. Unlike many other dimensionless numbers that we find in nature, such as the number of particles in the Universe, the fine structure constant represents a small number. This fact has led P.A.M. Dirac to formulate his "large number hypothesis" (Dirac 1937), where he constructed small dimensionless numbers from known physical constants assuming these are the fundamental parameters. One of these small numbers is the age of the Universe in atomic units divided by the electromagnetic force between an electron and a proton measured in units of their gravitational force and was believed to be $\approx 3$ in 1937 . Following this hypothesis, the gravitational constant $G$ or any other constant that appears in the construction of these small numbers should vary in time as the Universe expands. This was the first alternative theory assuming time-dependent coupling constants after Einstein's General Relativity. Indeed, there is no theory yet that predicts the value of $\alpha$ to be stable or drifting so that there is no reason to expect one or the other behavior. Even though Dirac's estimated drift rate of $\alpha$ has been ruled out by repeated measurements, the general possibility of "variable constants" remains open.

Modern theories that go beyond the large number hypothesis which allow for the drift of fundamental constants rely on coupling between gravitation and other fundamental interactions. Attempts to unify gravity with electromagnetic, weak and strong interactions encounter severe difficulties. To build such a "theory of everything" it seems that one has to extend the number of dimensions of our usual space-time world. String theories may allow for temporal and spatial variation of the coupling constants that could be associated with cosmic dynamics. Some possible mechanisms that lead to a drift or spatial variations of the fundamental constants are discussed in Taylor and Veneziano (1988), Damour et al. (2002), Flambaum (2007) and Flambaum and Shuryak (2008). As of now there is not sufficient theoretical evidence to make any well-grounded prediction of the size of such variations. The effect, if existing at all, should be extremely small since the gravitational interaction seems to be almost decoupled at the low-energy limit. For this reason experimental research is the appropriate way to probe this type of new physics that goes beyond the standard model.

Concerning the time interval $\Delta t$ separating two measurements, there are two extreme classes of experiments: (i) astronomical or geological observations and (ii) high precision laboratory measurements. The investigation of absorption or emission lines of distant galaxies back illuminated by the white light of quasars at even larger distances takes advantage of the extremely long look back time of up to $10^{10}$ years while the relative sensitivity to the $\alpha$ variation reaches $10^{-6}$. In contrast to that, laboratory frequency comparisons are restricted to short time intervals of a few years but can be as sensitive if precision measurements with uncertainties of better than $10^{-15}$ are performed. Currently such a low uncertainty can only be realized by frequency measurements in the radio or optical domain. For this reason frequency comparisons of atomic, molecular or ionic transitions are used. The important advantages of laboratory experiments are: The variety of different systems that may be tested, the possibility to change parameters of the experiments in order to control systematic effects and the straightforward determination of the drift rates from the measured values. Modern precision frequency measurements deliver information about the stability of the to-date values of the constants, which can only be tested with laboratory measurements. At the same time only non-laboratory methods are sensitive to processes that occurred in the early Universe, which may be much larger than at present times. As both classes of experiments 
Fig. 1 Uncertainties of the microwave Cs ground state hyperfine splitting (diamonds) and optical frequency standards based on a variety of atoms and ions (circles)

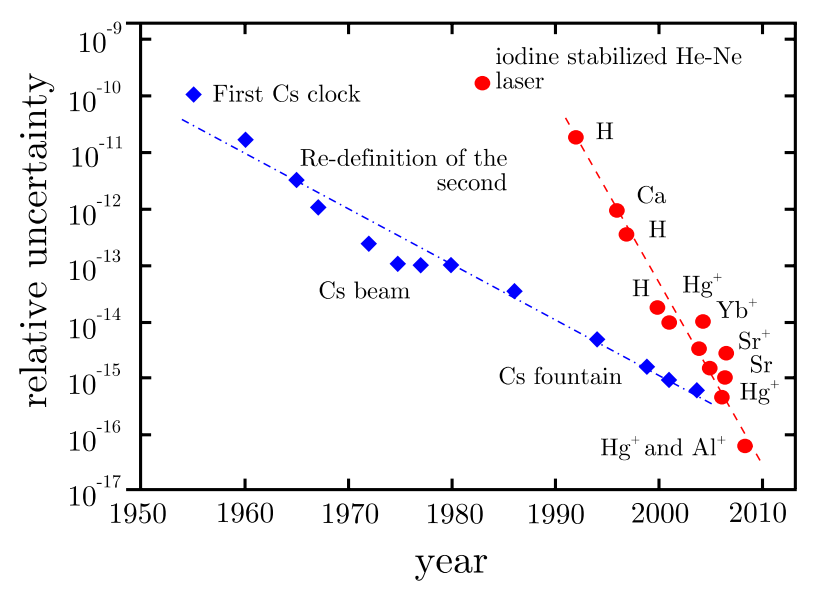

(i) and belong to different epochs, they supplement each other to get a more detailed view of the possible time variation of fundamental constants.

In 2000 J.K. Webb and co-workers introduced the many-multiplet method (Webb et al. 2001), which is an extension of the alkali-doublet method (Savedoff 1956; Minkowski and Wilson 1956), to extract the value of the fine structure constant from quasar absorption spectra. As the detected absorption lines emerged billions of light years away they conserve the value of $\alpha$ over that period of time. Application of the many-multiplet method to KECK/HIRES QSO data indicated that $\alpha$ was smaller by $\Delta \alpha / \alpha=(5.4 \pm 1.2) \times 10^{-6}$ about $10^{10}$ years ago (Webb et al. 2001). This $5 \sigma$ deviation from a non drifting value stimulated further investigation in the field. In 2003-2004 another set of astrophysical data obtained by the Very Large Telescope was analyzed independently using the same approach (Chand et al. 2004; Quast et al. 2004). The conclusion was that $\alpha$ was stable within $|\Delta \alpha / \alpha|<10^{-6}$ in the past. Meanwhile M.T. Murphy and co-workers pointed out some possible flaws in the data evaluation (Murphy et al. 2008) so that astrophysical data remain contradictory (see also Varshalovich et al. 2003). Unlike laboratory measurements astrophysical data analysis strongly relies on cosmological evolution, i.e. expansion, isotopic abundances, magnetic field distribution, etc. which are also debated.

Laboratory frequency measurements have become competitive very recently in terms of sensitivity to a possible variation of $\alpha$ in the present epoch. Figure 1 summarizes the progress achieved during the last decades in the field of optical frequency measurements (only a few are selected for the plot). For comparison, the progress in microwave frequency standards used for realization of the SI second is shown in the same plot. Improvements of the last years have been due to new ultra-cold atomic samples, laser stabilization techniques as well as breakthroughs in optical frequency measurements so that relative uncertainties in the optical domain are approaching $10^{-17}$.

With the introduction of frequency combs (see Sect. 2.2) high-precision optical frequency measurements became a routine procedure, readily available for a broad scientific community (Holzwarth et al. 2000; Udem et al. 2002). Repeated frequency measurements of some atomic transitions allowed to tighten the upper limit for the variation of frequency ratios. The latter can be used in a variety of fundamental tests, including the search for the variation of $\alpha$. Optical frequency measurements from 2000 and 2003 in ytterbium (Peik et al. 2004) and mercury (Bize et al. 2003) ions as well as in atomic hydrogen (Fischer et al. 2004) allowed to impose a model-independent restriction of $\dot{\alpha} / \alpha=(-0.9 \pm 2.9) \times 10^{-15} \mathrm{yr}^{-1}$ 

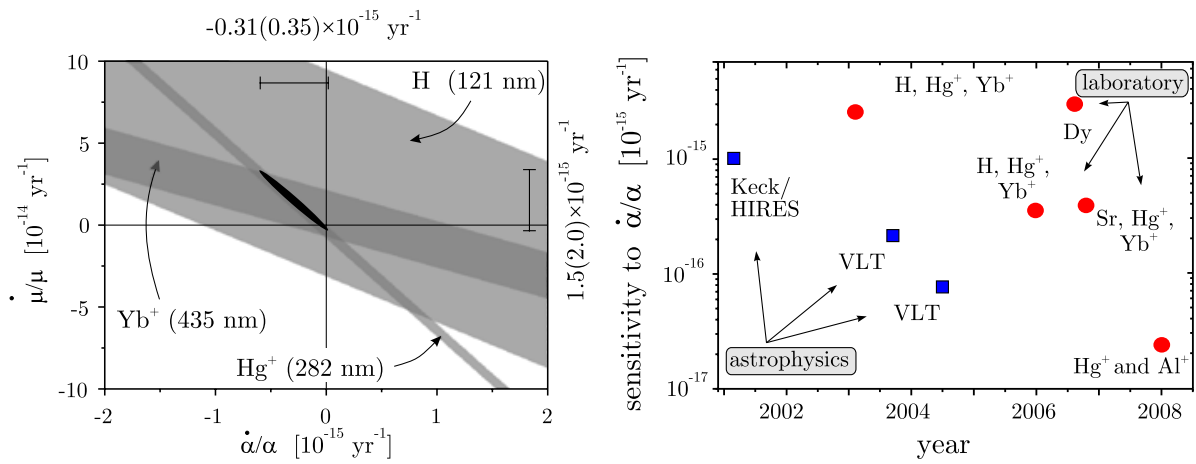

Fig. 2 (Left) - Illustration of the laboratory method for detecting possible variations of the fine structure constant $\alpha$. The method is based on combinations of absolute frequency measurements in different atomic systems (Peik et al. 2004; Fischer et al. 2004; Fortier et al. 2007). Here $\mu$ is the reduced magnetic moment of the ${ }^{133}$ Cs nucleus. (Right) - Comparison of sensitivities of astrophysical and laboratory methods for a presumed linear drift of $\alpha$

(see Fig. 2, left). The sensitivity of this test was already competitive to the sensitivity of the KECK/HIRES data analysis (Webb et al. 2001) (Fig. 2, right) which was the most sensitive analysis from astrophysical observations at that time.

The laboratory method is based on optical frequency measurements and allowed to increase the sensitivity of probing the possible $\alpha$ variation by an order of magnitude already in 2005 (Peik et al. 2006; Blatt et al. 2008). Thus far the lowest limit on the present drift rate of $\alpha$ has been obtained by T. Rosenband and co-workers at NIST (USA) by direct comparison of optical clock transitions in mercury and aluminum ions via a frequency comb (Rosenband et al. 2008; Lorini et al. 2008). Their result reads $\dot{\alpha} / \alpha=(-1.6 \pm 2.3) \times 10^{-17} \mathrm{yr}^{-1}$ and is an order of magnitude more accurate than astrophysical observations albeit at a different epoch.

In what follows we present modern techniques used for spectroscopy and frequency measurement of narrow optical transitions (Sect. 2), discuss the model-independent laboratory method for restricting the variation of $\alpha$ (Sect. 3) and point out some perspectives opened by optical frequency metrology for astrophysics (Sect. 4).

\section{Precision Optical Spectroscopy and Optical Frequency Measurements}

The principle of modern optical frequency measurement is presented in Fig. 3. A laser is tuned to the wavelength of a narrow metrological transition (usually referred to as a "clock transition") in an atomic, ionic or molecular sample. Most commonly, the laser frequency is stabilized by active feedback to a transmission peak of a well isolated optical cavity ("reference cavity") which allows to achieve sub-hertz spectral line width of the interrogating laser. Some recent advances in laser stabilization techniques will be described in Sect. 2.1. The laser frequency is then scanned across the transition which allows to find the line center $\omega_{0}$ using an appropriate line shape model. The measured transition quality factor can reach $10^{15}$ which provides extremely high resolution. To obtain the transition frequency the beat note $\omega_{\text {beat }}$ between the laser and one of the modes of a stabilized frequency comb is measured with the help of a frequency counter. Details of this type of measurement are presented in Sect. 2.2. If the comb is stabilized to a primary frequency reference (i.e. a Cs atomic clock), 
Fig. 3 Setup for the measurement an optical transition frequency in an atomic sample with the help of an optical frequency comb

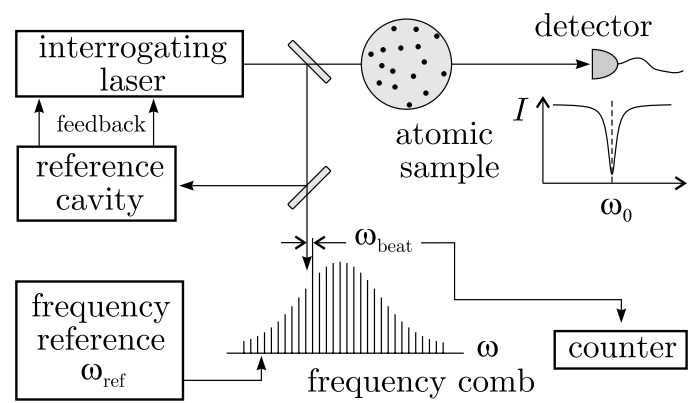

the measurement presented in Fig. 3 will yield the absolute frequency of the optical transition. Absolute frequency measurements allow a comparison of different results obtained at laboratories all over the world. An example of such a comparison is given in Sect. 3.1. On the other hand, if the comb is stabilized with the help of some other reference, which can be e.g. another optical frequency, the measurement will yield the ratio $\omega_{0} / \omega_{\text {ref }}$. One can thus compare transition frequencies in different atomic samples avoiding time-consuming absolute frequency measurements and avoiding the additional uncertainty introduced by primary frequency standards (Fig. 1).

\subsection{Laser Stabilization}

Interrogation of a clock transition in an atomic sample requires a narrow-band laser source. Due to vibrations, fluctuations of the pump intensity as well as some intrinsic noise sources (e.g. the excessive phase noise in semiconductor lasers, Petermann 1979; Henry 1982), the typical laser line width turns out to be many orders of magnitude broader than the SchawlowTownes limit (Schawlow and Townes 1958). A passive isolation of the laser resonator itself is not sufficient to suppress these noise sources so that active stabilization to an external reference cavity is implemented.

The reference cavity should be well isolated from the environment by placing it in a separate vibrationally and thermally stabilized vacuum chamber. If the laser frequency is stabilized to the transmission peak of such a cavity, e.g. by means of the Pound-Drever-Hall technique (Drever et al. 1983), the laser frequency fluctuations $\delta v$ will be directly coupled to the fluctuations of the cavity length $\delta l$ according to $\delta v / v=\delta l / l$, where $v$ is the laser frequency, and $l$ is the cavity length. If one desires to achieve $\delta v=1 \mathrm{~Hz}$ using a cavity of $l=10 \mathrm{~cm}$, the distance between the mirrors should remain constant at the level of $10^{-16} \mathrm{~m}$, which is a fraction of the proton radius!

The first demonstration of a sub-Hz laser line width by B.C. Young and co-workers in 1999 made use of a heavy optical bench suspended with rubber tubes from the lab ceiling (Young et al. 1999) for vibration isolation. In the meantime cavity designs and mountings emerged where deformations due to vibrations do not change the critical length that separates the mirrors (Notcutt et al. 2005; Nazarova et al. 2006). This has not only led to much more compact setups but also to a number of laser sources successfully stabilized to sub-hertz level (Stoehr et al. 2006; Ludlow et al. 2007). In our laboratory we use vertically mounted Fabry-Pérot (FP) cavities, with a spacer design from A.D. Ludlow and coworkers (2007) and reach $40 \mathrm{~dB}$ suppression of vertical vibration sensitivity (Alnis et al. 2008).

The principle of such a cavity mounting is shown in the left hand side of Fig. 4. The cavity spacer is suspended at its midplane such that the influence of vibration induced ver- 

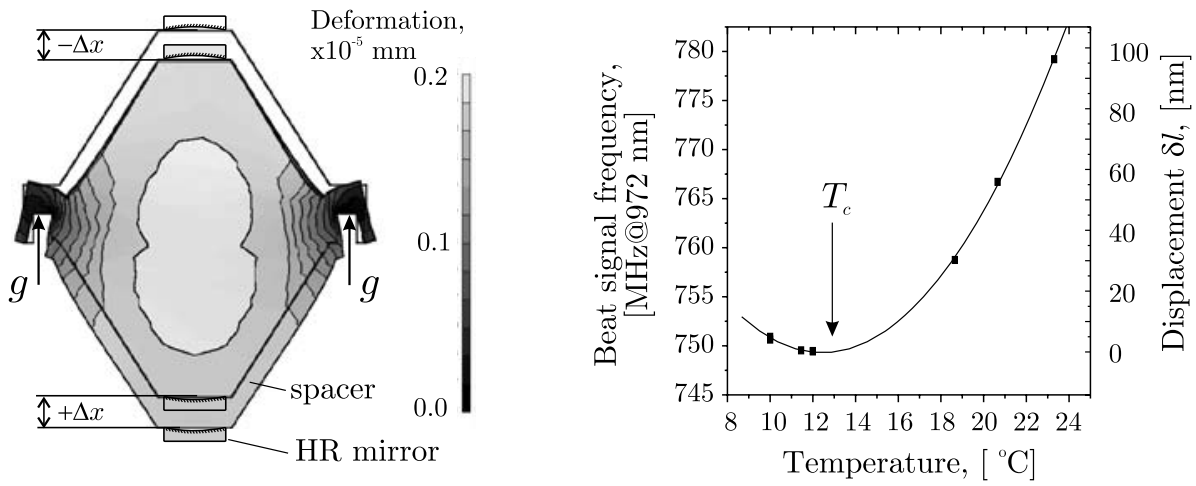

Fig. 4 (Left)-Deformations of a vertically mounted Fabry-Pérot (FP) cavity under the influence of vertical acceleration of $1 \mathrm{~g}$ determined with finite elements analysis. The mounting is such that the compression of upper part $-\Delta x$ is compensated by the stretch of the lower part $+\Delta x$ maintaining the critical distance of the mirrors. (Right) —Determination of the zero-expansion temperature $T_{c}$ by measuring the beat note frequency between the FP cavity and the laser stabilized to the second FP cavity maintained at a constant temperature. At $T_{c}$ the cavity length $l$ reaches the minimum

tical acceleration to the mirror separation is significantly suppressed. This makes the setup virtually immune for vertical vibrations that are more difficult to suppress than horizontal ones. If such a cavity is placed on a vibration-isolated platform, the acoustic and seismic vibrations from the environment have no detectable influence on the cavity frequency.

Another issue that affects the stability for averaging times larger than several seconds is the dimensional stability due to temperature variations. Certain glass ceramics can be made with very low thermal expansion and the one made by Corning is called ultra low expansion glass (ULE). ULE is a Titania-doped silicate glass that has a specified thermal expansion minimum at some temperature $T_{c}$ around room temperature according to

$$
\delta l / l \sim 10^{-9}\left(T-T_{c}\right)^{2},
$$

where $T$ is the cavity temperature. To reduce the quadratic dependence the temperature of the material should be stabilized as close as possible to $T_{c}$. Unfortunately, the measured composite thermal stationary point of the cavity resonance frequency usually ends up below room temperature (Fox 2008) (Fig. 4, right). This poses a problem because cooling of the vacuum chamber is more difficult than heating as water condensation on the windows prevents coupling of laser light into the chamber. The problem was solved by cooling the FP cavity directly in the vacuum chamber by Peltier elements (Alnis et al. 2008).

This type of vibration- and thermal compensation allows to set up extremely stable and compact laser sources. For example, the diode lasers operating at $972 \mathrm{~nm}$ designed for twophoton spectroscopy of the $1 S-2 S$ clock transition in atomic hydrogen are characterized as shown in Fig. 5. Two nearly identical systems have been built in our lab with one FP cavity maintained near $T_{c}$ of its resonance frequency, while the other stabilized to a temperature $25^{\circ} \mathrm{C}$ above that point. Both lasers demonstrate excellent short-time stability (up to $10 \mathrm{~s}$ ) approaching the thermal noise limit of $10^{-15}$ which is set by the Brownian particle motion on the mirror surfaces. Concerning the long-term stability, the FP cavity at $T_{c}$ demonstrates a much better performance since it is much less influenced by ambient temperature fluctuations. It possesses a linear drift of about $+50 \mathrm{mHz} / \mathrm{s}$ mainly caused by ULE aging, while its resonance frequency deviates from that linear drift by only $\pm 10 \mathrm{~Hz}$ on a time scale of $10 \mathrm{hrs}$. 

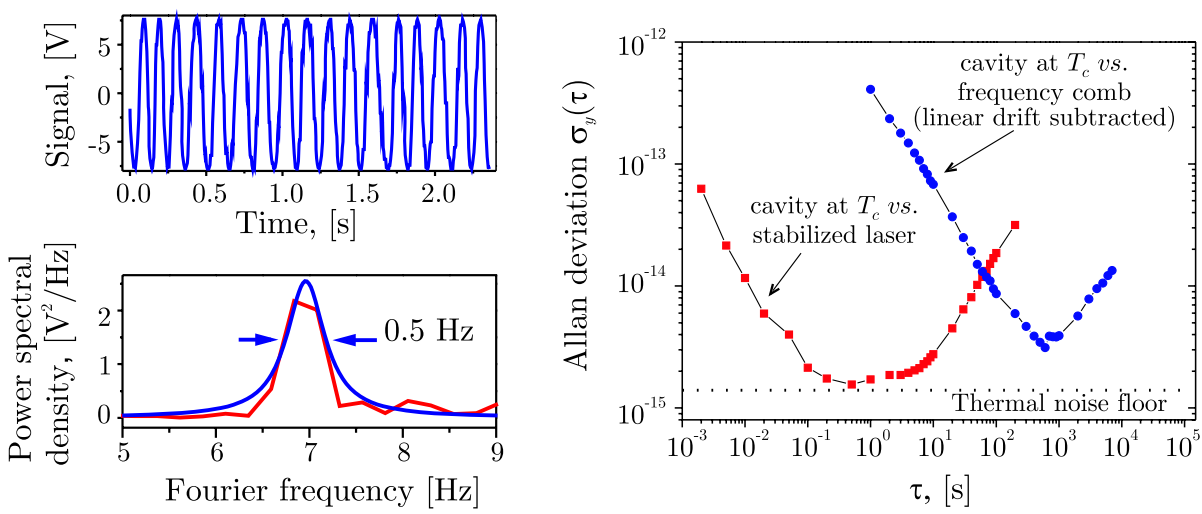

Fig. 5 (Left)-Oscillogram of the beat note signal between two diode lasers locked to two independent vertically mounted FP cavities, its Fourier transformation and Lorentzian fit. (Right)_Allan deviation of the beat note signal from the left part of the figure (squares). The cavity kept at $T_{C}$ is extremely stable as can be seen from its absolute frequency measurement performed with an optical frequency comb (see next section) which was referenced to a hydrogen maser (circles)

Excellent spectral characteristics and small size of the setup allow to use such lasers in the most demanding applications of frequency metrology. Possible routes to overcome the thermal noise limit are currently being discussed and may result in further improvements of the laser spectral purity.

\subsection{Ultra-Short Pulse Lasers and Frequency Combs}

Frequency can be measured with by far the highest precision of all physical quantities. In the radio frequency domain (say up to $100 \mathrm{GHz}$ ), frequency counters have existed for a long time. Almost any of the most precise measurements in physics have been performed with such a counter that uses an atomic clock as a time base. To extend this accurate technique to higher frequencies, so called harmonic frequency chains have been constructed since the late 1960ies (Hocker et al. 1967; Evenson et al. 1973). Because of the large number of steps necessary to build a long harmonic frequency chain, it was not before 1995 when visible laser light was first referenced phase coherently to a cesium atomic clock using this method (Schnatz et al. 1996).

The disadvantage of these harmonic frequency chains was not only that they could easily fill several large laser laboratories at once, but that they could be used to measure a single optical frequency only. Even though mode locked lasers for optical frequency measurements have been used in rudimentary form in the late 1970ies (Eckstein et al. 1978), this method became only practical with the advent of femtosecond (fs) mode locked lasers. Such a laser necessarily emits a very broad spectrum, comparable in width to the optical carrier frequency.

In the frequency domain a train of short pulses from a femtosecond mode locked laser is the result of a phase coherent superposition of many continuous wave (cw) longitudinal cavity modes. These modes at $\omega_{n}$ form a series of frequency spikes that is called a frequency comb. As has been shown, the modes are remarkably uniform, i.e. the separation between adjacent modes is constant across the frequency comb (Holzwarth et al. 2000; Udem et al. 1999; Diddams et al. 2002; Ma et al. 2004). This strictly regular arrangement is the most 
important feature used for optical frequency measurement and may be expressed as:

$$
\omega_{n}=n \omega_{r}+\omega_{C E} .
$$

Here the mode number $n$ of some $10^{5}$ may be enumerated such that the frequency offset $\omega_{C E}$ lies in between 0 and $\omega_{r}=2 \pi / T$. The mode spacing is thereby identified with the pulse repetition rate, i.e. the inverse pulse repetition time $T$. With the help of that equation two radio frequencies $\omega_{r}$ and $\omega_{C E}$ are linked to the optical frequencies $\omega_{n}$ of the laser. For this reason mode locked lasers are capable to replace the harmonic frequency chains of the past.

To derive the frequency comb properties (Reichert et al. 1999) as detailed by (2), it is useful to consider the electric field $E(t)$ of the emitted pulse train. We assume that the electric field $E(t)$, measured for example at the lasers output coupling mirror, can be written as the product of a periodic envelope function $A(t)$ and a carrier wave $C(t)$ :

$$
E(t)=A(t) C(t)+c . c .
$$

The envelope function defines the pulse repetition time $T=2 \pi / \omega_{r}$ by demanding $A(t)=$ $A(t-T)$. The only thing about dispersion that should be added for this description, is that there might be a difference between the group velocity and the phase velocity inside the laser cavity. This will shift the carrier with respect to the envelope by a certain amount after each round trip. The electric field is therefore in general not periodic with $T$. To obtain the spectrum of $E(t)$ the Fourier integral has to be calculated:

$$
\tilde{E}(\omega)=\int_{-\infty}^{+\infty} E(t) e^{i \omega t} d t
$$

Separate Fourier transforms of $A(t)$ and $C(t)$ are given by:

$$
\tilde{A}(\omega)=\sum_{n=-\infty}^{+\infty} \delta\left(\omega-n \omega_{r}\right) \tilde{A}_{n} \quad \text { and } \quad \tilde{C}(\omega)=\int_{-\infty}^{+\infty} C(t) e^{i \omega t} d t
$$

A periodic frequency chirp imposed on the pulses is accounted for by allowing a complex envelope function $A(t)$. Thus the "carrier" $C(t)$ is defined to be whatever part of the electric field that is non-periodic with $T$. The convolution theorem allows us to calculate the Fourier transform of $E(t)$ from $\tilde{A}(\omega)$ and $\tilde{C}(\omega)$ :

$$
\tilde{E}(\omega)=\frac{1}{2 \pi} \int_{-\infty}^{+\infty} \tilde{A}\left(\omega^{\prime}\right) \tilde{C}\left(\omega-\omega^{\prime}\right) d \omega^{\prime}+c . c .=\frac{1}{2 \pi} \sum_{n=-\infty}^{+\infty} \tilde{A}_{n} \tilde{C}\left(\omega-n \omega_{r}\right)+c . c .
$$

The sum represents a periodic spectrum in frequency space. If the spectral width of the carrier wave $\Delta \omega_{c}$ is much smaller than the mode separation $\omega_{r}$, it represents a regularly spaced comb of laser modes just like (2), with identical spectral line shapes. If $\tilde{C}(\omega)$ is centered at say $\omega_{c}$, then the comb is shifted by $\omega_{c}$ from containing only exact harmonics of $\omega_{r}$. The frequencies of the mode members are calculated from the mode number $n$ (Udem et al. 2002; Eckstein et al. 1978; Reichert et al. 1999):

$$
\omega_{n}=n \omega_{r}+\omega_{c} .
$$

The measurement of the $\omega_{c}$ as described below (see also Holzwarth et al. 2000; Reichert et al. 1999; Diddams et al. 2000; Udem et al. 1999, 2002) usually yields a value modulo $\omega_{r}$, 
Fig. 6 Consecutive un-chirped pulses $(A(t)$ is real) with carrier frequency $\omega_{c}$ and the corresponding spectrum (not to scale). Because the carrier propagates with a different velocity within the laser cavity than the envelope (with phaseand group velocity respectively), the electric field does not repeat itself after one round trip. A pulse-to-pulse phase shift $\Delta \varphi$ results in an offset frequency of $\omega_{C E}=\Delta \varphi / T$. The mode spacing is given by the repetition rate $\omega_{r}$. The width of the spectral envelope is given by the inverse pulse duration up to a factor of order unity that depends on the pulse shape

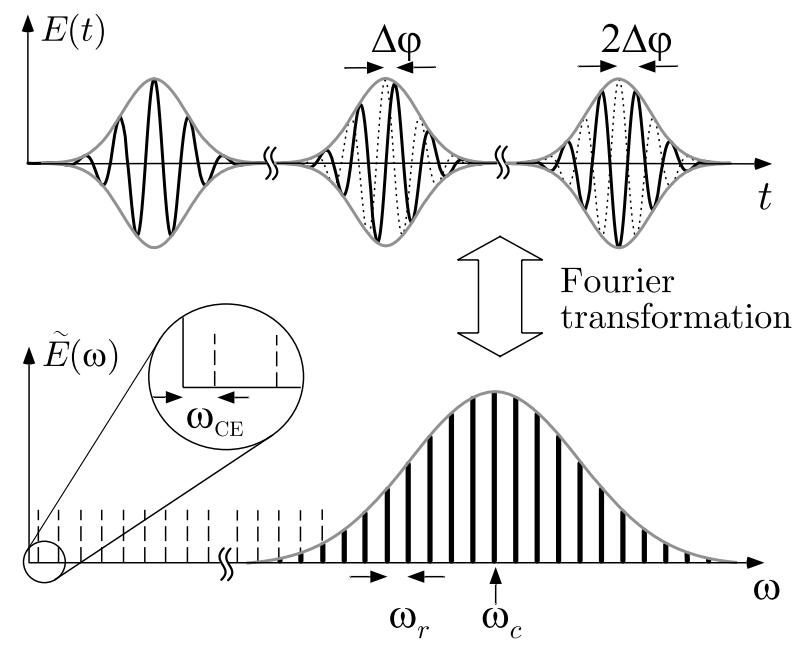

so that renumbering the modes will restrict the offset frequency to smaller values than the repetition frequency and (2) and (7) are identical.

If the carrier wave is monochromatic $C(t)=e^{-i \omega_{c} t-i \varphi}$, its spectrum will be $\delta$-shaped and centered at the carrier frequency $\omega_{c}$. The individual modes are also $\delta$-functions $\tilde{C}(\omega)=$ $\delta\left(\omega-\omega_{c}\right) e^{-i \varphi}$. The frequency offset (7) is identified with the carrier frequency. According to (3) each round trip will shift the carrier wave with respect to the envelope by $\Delta \varphi=\arg (C(t-T))-\arg (C(t))=\omega_{c} T$ so that the frequency offset may also be identified by $\omega_{C E}=\Delta \varphi / T$ (Udem et al. 2002; Eckstein et al. 1978; Reichert et al. 1999). In a typical laser cavity this pulse-to-pulse carrier-envelope phase shift is much larger than $2 \pi$, but measurements usually yield a value modulo $2 \pi$. The restriction $0 \leq \Delta \varphi \leq 2 \pi$ is synonymous with the restriction $0 \leq \omega_{C E} \leq \omega_{r}$ introduced above. Figure 6 sketches this situation in the time domain for a chirp free pulse train.

\subsubsection{Extending the Frequency Comb}

The spectral width of a pulse train emitted by a fs laser can be significantly broadened in a single mode fiber (Agrawal 2001) by self phase modulation. Assuming a single mode carrier wave, a pulse that has propagated the length $L$ acquires a self induced phase shift of

$$
\Phi_{N L}(t)=-n_{2} I(t) \omega_{c} L / c,
$$

where the pulse intensity is given by $I(t)=\frac{1}{2} c \varepsilon_{0}|A(t)|^{2}$. For fused silica the non-linear Kerr coefficient $n_{2}$ is comparatively small but almost instantaneous even on the time scale of $\mathrm{fs}$ pulses. This means that different parts of the pulse travel at different speed. The result is a frequency chirp across the pulse without affecting its duration. The pulse is no longer at the Fourier limit so that the spectrum is much broader than the inverse pulse duration where the extra frequencies are determined by the time derivative of the self induced phase shift $\dot{\Phi}_{N L}(t)$. Therefore pure self-phase modulation would modify the envelope function in (3) according to

$$
A(t) \longrightarrow A(t) e^{i \Phi_{N L}(t)}
$$




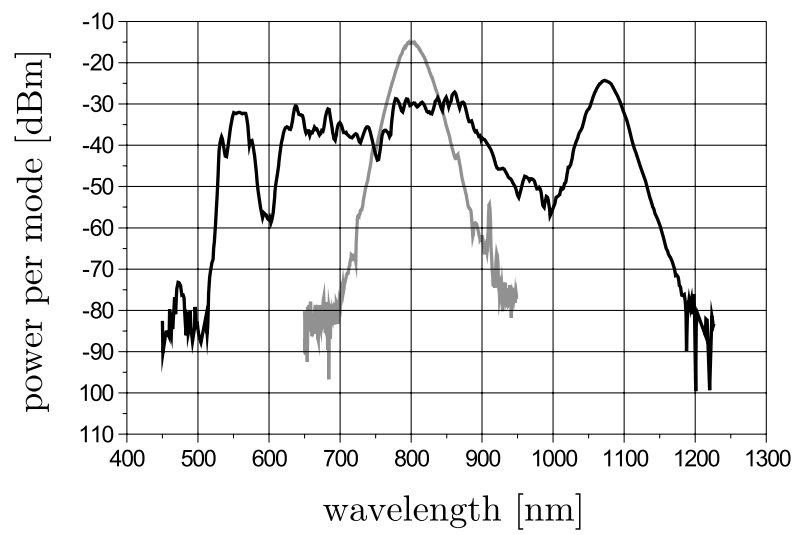

Fig. 7 Power per mode of the frequency comb on a logarithmic scale $(0 \mathrm{dBm}=1 \mathrm{~mW})$. The lighter $30 \mathrm{~nm}$ $(14 \mathrm{THz}$ at $-3 \mathrm{~dB}$ ) wide spectrum displays the laser intensity and the darker octave spanning spectrum (532 $\mathrm{nm}$ through $1064 \mathrm{~nm}$ ) is observed after spectral broadening in a $30 \mathrm{~cm}$ microstructured fiber (Knight et al. 1996). The laser was operated at $\omega_{r}=2 \pi \times 750 \mathrm{MHz}$ (modes not resolved) with $25 \mathrm{fs}$ pulse duration. An average power of $180 \mathrm{~mW}$ was coupled through the microstructure fiber (Holzwarth et al. 2001)

Because $\Phi_{N L}(t)$ has the same periodicity as $A(t)$ the comb structure of the spectrum is maintained and the derivations (6) remain valid because periodicity of $A(t)$ was the only assumption made. An optical fiber is most appropriate for this process because it can maintain the necessary small focus area over a virtually unlimited length. In practice, however, other pulse reshaping mechanism, both linear and non-linear, are present so that the above explanation might be too simple.

A microstructured fiber uses an array of submicron-sized air holes that surround the fiber core and run the length of a silica fiber to obtain a desired effective dispersion (Knight et al. 1996). This can be used to maintain the high peak power over an extended propagation length and to significantly increase the spectral broadening. With these fibers it became possible to broaden low peak power, high repetition rate lasers to beyond one optical octave as shown in Fig. 7.

Another class of frequency combs that can stay in lock for longer times are fs fiber lasers (Nelson et al. 1997). The most common type is the erbium doped fiber laser that emits within the telecom band around $1550 \mathrm{~nm}$. For this reason advanced and cheap optical components are available to build such a laser. The mode locking mechanism is similar to the Kerr lens method, except that non-linear polarization rotation is used to favor the pulsed high peak intensity operation. Up to a short free space section that can be build very stable, these lasers have no adjustable parts. Continuous stabilized operation for many hours (Kubina et al. 2005; Adler et al. 2004) has been reported. The Max-Planck Institut für Quantenoptik in Garching (Germany) operates a fiber based self referenced frequency comb that stays locked without interruption for months.

\subsubsection{Self-Referencing}

The measurement of $\omega_{C E}$ fixes the position of the whole frequency comb and is called selfreferencing. The method relies on measuring the frequency gap between different harmonics derived from the same laser or frequency comb. The simplest approach is to fix the absolute position of the frequency comb by measuring the gap between $\omega_{n}$ and $\omega_{2 n}$ of modes taken 

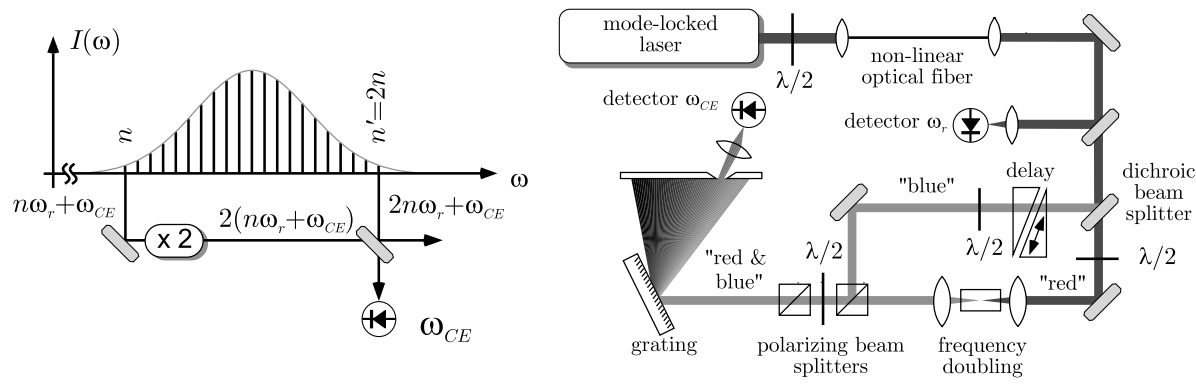

Fig. 8 (Left) - The principle of the $f-2 f$ self referencing relies on detecting a beat note at ${ }_{C} C E$ between the frequency doubled "red" wing $2\left(n \omega_{r}+\omega_{C E}\right)$ of the frequency comb and the "blue" modes at $2 n \omega_{r}+\omega_{C E}$. (Right) - More detailed layout of the self referencing scheme. See text for details

directly from the frequency comb (Holzwarth et al. 2000; Udem et al. 2002; Diddams et al. 2000; Reichert et al. 2000). In this case the carrier-envelope offset frequency $\omega_{C E}$ is directly produced by beating the frequency doubled ${ }^{1}$ red wing of the comb $2 \omega_{n}$ with the blue side of the comb at $\omega_{2 n}: 2 \omega_{n}-\omega_{n^{\prime}}=\left(2 n-n^{\prime}\right) \omega_{r}+\omega_{C E}=\omega_{C E}$ where again the mode numbers $n$ and $n^{\prime}$ are chosen such that $\left(2 n-n^{\prime}\right)=0$. This approach requires an octave spanning comb, i.e. a bandwidth of $375 \mathrm{THz}$ if centered at the titanium-sapphire gain maximum at $800 \mathrm{~nm}$.

Figure 8 sketches the $f-2 f$ self referencing method. The spectrum of a mode locked laser is first broadened to more than one optical octave with an optical fiber. A broad band $\lambda / 2$ wave plate allows to choose the polarization with the most efficient spectral broadening. After the fiber a dichroic mirror separates the infrared ("red") part from the green ("blue"). The former is frequency doubled in a non-linear crystal and reunited with the green part to create a wealth of beat notes, all at $\omega_{C E}$. These beat notes emerge as frequency difference between $2 \omega_{n}-\omega_{2 n}$ according to (2) for various values of $n$. The number of contributing modes is given by the phase matching bandwidth $\Delta v_{p m}$ of the doubling crystal and can easily exceed $1 \mathrm{THz}$.

As described, both degrees of freedom $\omega_{r}$ and $\omega_{C E}$ of the frequency comb can be measured up to a sign in $\omega_{C E}$ that will be discussed below. For stabilization of these frequencies, say relative to a radio frequency reference, it is also necessary to control them. Again the repetition rate turns out to be simpler. Mounting one of the laser's cavity mirrors on a piezo electric transducer allows to control the pulse round trip time. Controlling the carrier envelope frequency requires some effort. Any laser parameter that has a different influence on the cavity round trip phase delay and the cavity round trip group delay may be used to change $\omega_{C E}$ (Haus and İppen 2001). Experimentally it turned out that the energy of the pulse stored inside the mode locked laser cavity has a strong influence on $\omega_{C E}$. To phase lock the carrier envelope offset frequency $\omega_{C E}$, one can therefore control the laser power through its energy source (pump laser).

\subsubsection{Frequency Conversions}

Given the above we conclude that the frequency comb may serve as a frequency converter between the optical and radio frequency domains allowing to perform the following phase coherent operations:

\footnotetext{
${ }^{1}$ It should be noted that this does not simply mean the doubling of each individual mode, but the general sum frequencies generation of all modes. Otherwise the mode spacing, and therefore the repetition rate, would be doubled as well.
} 
- convert a radio frequency into an optical frequency. In this case both $\omega_{r}$ and $\omega_{C E}$ from (2) are directly locked to the radio frequency source.

- convert an optical frequency into a radio frequency. In this case the frequency of one of the comb modes $\omega_{n}$ is locked to a clock laser while the carrier envelope frequency $\omega_{C E}$ is phase locked to $\omega_{r}$. The repetition rate will then be used as the countable clock output.

- convert an optical frequency to another optical frequency, i.e. measuring optical frequency ratios. In this case the comb is stabilized to one of the lasers as described in the second case, but instead of measuring $\omega_{r}$ one measures the beat note frequency between another laser and its closest comb mode $\omega_{n}^{\prime}$.

\subsection{Frequency Measurement of the $1 S-2 S$ Transition in Atomic Hydrogen}

One of the first optical frequency measurements performed with an optical frequency comb was the $1 S-2 S$ transition frequency in atomic hydrogen in our lab. During the last decades precision spectroscopic experiments on hydrogen and deuterium atoms have yielded new accurate values for the Rydberg constant (Biraben et al. 2001), the ground-state Lamb shift (Weitz et al. 1994), the deuteron structure radius (Huber et al. 1998), and the $2 S$ hyperfine structure (Kolachevsky et al. 2004a, 2004b). Accurate optical frequency measurements allow for sensitive tests of quantum electrodynamics (QED), which are based on comparisons between experimental values and results from corresponding QED calculations (for review see Eides et al. 2001; Karshenboim and Ivanov 2002a, 2002b).

To measure the frequency $\omega_{L}$ of the continuous wave (cw) interrogation laser $(486 \mathrm{~nm})$ that drives the $1 S-2 S$ transition, a beat note $\omega_{b}$ with a stabilized frequency comb is generated (see Fig. 8). For this purpose the beam of the $\mathrm{cw}$ laser is spatially overlapped with the beam that contains the frequency comb and guided to a photo detector. The frequency of the interrogation laser is then given by

$$
\omega_{L}=n \omega_{r} \pm \omega_{C E} \pm \omega_{b}
$$

The signs can be determined by introducing small changes to $\omega_{r}$ and $\omega_{C E}$ and observing the corresponding shift in $\omega_{b}$. This uniquely fixes both signs if $\omega_{L}$ is held fixed during this test. The mode number $n$ may be determined by a coarse measurement say with a highresolution wave meter, by re-measuring with different $\omega_{r}$ or by comparison with previous results of lower accuracy.

In 1999 the absolute frequency measurement resulted in a relative uncertainty of 1.8 parts in $10^{14}$ (Niering et al. 2000). In 2003 this measurement has been repeated and the results of both campaigns are shown in Fig. 9. In both cases a transportable Cs atomic fountain clock (FOM) from LNM-SYRTE Paris (Santarelli et al. 1999) has been transported to our lab at Garching. Its accuracy has been evaluated to $8 \times 10^{-16}$, but during the experiments a verification at the level of $2 \times 10^{-15}$ only has been performed which is still one order of magnitude better than required for the $1 S-2 S$ transition. Unfortunately, the uncertainty of the 2003 measurement remained nearly the same due to an excessive day-to-day scatter. A further improvement of the accuracy is expected by $1 S-2 S$ spectroscopy on hydrogen atoms decelerated magnetically from a supersonic beam (Vanhaecke et al. 2007; Narevicius et al. 2008).

The measurements allowed not only to determine the absolute frequency of the transition, but also to set an upper limit of the difference of $(-29 \pm 57) \mathrm{Hz}$ between the measurements that are 44 months apart. This is equivalent to a fractional time variation of the ratio $f_{\mathrm{H}} / f_{\mathrm{Cs}}$ equal to $(-3.2 \pm 6.3) \times 10^{-15} \mathrm{yr}^{-1}$, where the ground state hyperfine splitting of Cs-133, 
Fig. 9 Absolute frequency measurements of the $1 S-2 S$ transition in atomic hydrogen

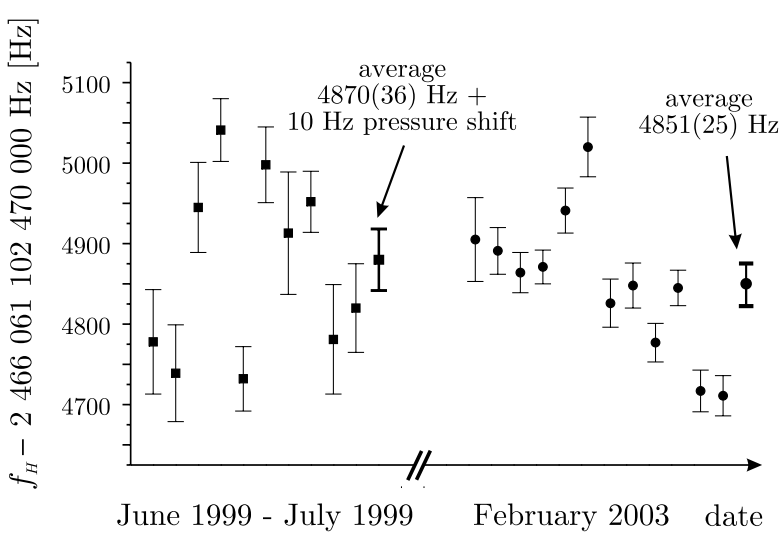

which is used as a reference in these measurements, is given by $f_{\mathrm{Cs}}$. This limit on the temporal variation of the absolute optical frequency opens the possibility to derive an upper limit of the variation of $\alpha$ as detailed in the next section.

\section{High-Precision Laboratory Measurements and Variation of the Fine Structure Constant}

So far all laboratory measurements of the drift rates of fundamental constants are based on comparisons of electromagnetic transitions that depend in a different way on these constants. The non-relativistic scalings of gross-, fine- and hyperfine transitions in atoms, ions and molecules are summarized in Table 1. A first order theory is sufficient here, as none of the drifts have been detected yet with small relative uncertainty. To evaluate the possible drift of $\alpha$ one measures a frequency ratio of two transitions. Pioneering astrophysical measurements (Savedoff 1956; Minkowski and Wilson 1956) used that method which is now called the "alkali-doublet method".

In a real situation the values in that table need to be multiplied with a relativistic correction $F_{\text {rel }}(Z \alpha)$ that depends only on the fine structure constant and may be determined from relativistic Hartree-Fock calculations. For hyperfine transitions in alkali atoms there exists an approximate expression for the relativistic correction called the Casimir correction (Casimir 1963) which reads as

$$
F_{\text {rel }}(Z \alpha)=\frac{3}{\lambda\left(4 \lambda^{2}-1\right)}, \quad \text { where } \lambda \equiv \sqrt{1-(Z \alpha)^{2}} .
$$

For heavy atomic systems the correction to the hyperfine levels $F_{\text {rel }}(Z \alpha)$ differs significantly from 1 (e.g. $F_{\text {rel }}=1.39$ for Cs) so that the sensitivity for $\alpha$ variations may be expressed as:

$$
L_{\alpha}^{(\mathrm{HFS})} \equiv \alpha \frac{\partial}{\partial \alpha} \ln \left[F_{\mathrm{rel}}(Z \alpha)\right]=(Z \alpha)^{2} \frac{12 \lambda^{2}-1}{\lambda^{2}\left(4 \lambda^{2}-1\right)} .
$$

For the Cs ground state hyperfine splitting this equals $L_{\alpha}^{(\mathrm{HFS})}(\mathrm{Cs}) \approx 0.8$. As only ratios of frequencies can be determined in a real measurement, the sensitivity of any experiment will be given by the ratio of the sensitivity functions of the involved frequencies. Therefore the sensitivity function defined above is meaningless until it is referenced to another one. 
Table 1 Scaling factors for different atomic systems in the non-relativistic approximation. Here $R y$ is the Rydberg constant in hertz, $g_{\text {nucl }}$ is the nuclear $g$-factor, $\mu_{N}$ and $\mu_{B}$-nuclear and Bohr magnetons respectively, $m_{e}$ and $m_{p}$-electron and proton mass respectively. In the relativistic case, i.e. for heavier atoms, it is necessary to multiply the scalings with the relativistic correction $F_{\text {rel }}(Z \alpha)$ that depends only on the fine structure constant and may be determined from relativistic Hartree-Fock calculations

\begin{tabular}{lll}
\hline Sample & Transition & Scaling factor \\
\hline Atom, ion & gross structure & $R y$ \\
& fine structure & $\alpha^{2} R y$ \\
& hyperfine structure & $g_{\text {nucl }}\left(\mu_{N} / \mu_{B}\right) \alpha^{2} R y$ \\
Molecule & gross structure & $R y$ \\
& vibration structure & $\left(m_{e} / m_{p}\right)^{1 / 2} R y$ \\
& rotational structure & $\left(m_{e} / m_{p}\right) R y$ \\
\hline
\end{tabular}

For optical transition frequencies $f^{(\mathrm{opt})}$ no approximation such as the Casimir correction exists that would be useful for deriving the leading order dependence on the fine structure constant. For this reason relativistic Hartree-Fock calculations have been used. V.A. Dzuba and co-workers have expressed the results of their calculation in terms of the two parameters $q_{1}$ and $q_{2}$ according to:

$$
f^{(\mathrm{opt})}=f_{0}^{(\mathrm{opt})}+q_{1}\left[\left(\frac{\alpha}{\alpha_{0}}\right)^{2}-1\right]+q_{2}\left[\left(\frac{\alpha}{\alpha_{0}}\right)^{4}-1\right] .
$$

Here $f_{0}^{(\mathrm{opt})}$ and $\alpha_{0}$ are the present day (or laboratory) values of the optical transition frequency and the fine structure constant respectively. This equation was used to describe quasar absorption spectra but may be used for laboratory measurements. In this case $f_{0}^{(\mathrm{opt})}$ and $\alpha_{0}$ are also laboratory values but at different times. Results for the parameters $q_{1}$ and $q_{2}$ for various atoms and ions including some important optical clock transitions are published in Dzuba et al. (1999a, 1999b), Dzuba and Flambaum (2000). Only even powers in $\alpha$ enter the expansion (13) because the relativistic correction is proportional to $\sqrt{m_{e}^{2}+p^{2}}$, which contains even powers of electron momentum $p \sim Z \alpha$. Re-expressing Dzuba's notation in terms of the relativistic correction introduced above yields:

$$
L_{\alpha}^{(\mathrm{opt})} \equiv \alpha \frac{\partial}{\partial \alpha} \ln F_{\text {rel }}(Z \alpha)=\frac{2 q_{1}+4 q_{2}}{f_{0}^{(\mathrm{opt})}} .
$$

Table 2 lists a few values of this quantity adapted from Dzuba et al. (1999a, 1999b), Dzuba and Flambaum (2000) that are relevant for metrological transitions. Note, that for these calculations the value of $R y$ has been assumed to be fixed which imposes a constrain on the value of the product $m_{e} c^{2} \alpha^{2} / h$. Another way of interpreting this is by picking $R y$ as the unit of frequency. Using the same unit for all frequencies it will eventually drop out of all measurable quantities as only frequency ratios can be determined in practice. This will become more clear from the further analysis.

Comparing optical transitions with different relativistic corrections became a powerful instrument to set upper limits to the drift of fundamental constants. This method is widely used in astrophysical observations ("many-multiplet" method Webb et al. 2001) and in laboratory comparisons. An elegant realization of this method has been used by A. Cingöz et al. (2007), by utilizing different relativistic corrections of two nearly degenerate levels of opposite parity in neutral dysprosium. Monitoring the radio frequency transitions at $3.1 \mathrm{MHz}$ for 
Table 2 Sensitivity of relativistic corrections $F_{\text {rel }}(Z \alpha)$ to $\alpha$ for some atomic transitions according to Dzuba et al. (1999a, 1999b), Dzuba and Flambaum (2000)

\begin{tabular}{lllcc}
\hline$Z$ & Atom & Transition & $\lambda[\mathrm{nm}]$ & $L_{\alpha}^{(\text {opt })}$ \\
\hline 1 & $\mathrm{H}$ & $1 \mathrm{~s}_{1 / 2}\left(F=1, m_{F}= \pm 1\right) \rightarrow 2 \mathrm{~s} \mathrm{~S}_{1 / 2}\left(F^{\prime}=1, m_{F}^{\prime}= \pm 1\right)$ & 121 & 0 \\
20 & $\mathrm{Ca}$ & ${ }^{1} \mathrm{~S}_{0}\left(m_{J}=0\right) \rightarrow{ }^{3} \mathrm{P}_{1}\left(m_{J}=0\right)$ & 657 & 0.03 \\
49 & $\mathrm{In}^{+}$ & $5 \mathrm{~s}^{2}{ }^{1} \mathrm{~S}_{0} \rightarrow 5 \mathrm{~s} 5 \mathrm{p}^{3} \mathrm{P}_{0}$ & 237 & 0.21 \\
70 & $\mathrm{Yb}^{+}$ & $6 \mathrm{~s}^{2} \mathrm{~S}_{1 / 2}(F=0) \rightarrow 5 \mathrm{~d}^{2} \mathrm{D}_{3 / 2}(F=2)$ & 435 & 0.9 \\
80 & $\mathrm{Hg}^{+}$ & $5 \mathrm{~d}^{10} 6 \mathrm{~s}^{2} \mathrm{~S}_{1 / 2}(F=0) \rightarrow 5 \mathrm{~d}^{9} 6 \mathrm{~s}^{2}{ }^{2} \mathrm{D}_{5 / 2}\left(F^{\prime}=2, m_{F}^{\prime}=0\right)$ & 282 & -3.2 \\
\hline
\end{tabular}

${ }^{163}$ Dy and $325 \mathrm{MHz}$ for ${ }^{162}$ Dy during 8 months only, the authors set a stringent limit on the drift of the fine-structure constant of $\partial \ln (\alpha) / \partial t=(-2.7 \pm 2.6) \times 10^{-15} \mathrm{yr}^{-1}$ without any assumptions about the drift of other constants. In the next section we will describe how one can deduce a model-independent restriction to $\dot{\alpha}$ from different absolute optical frequency measurements.

\subsection{Upper Limit for the Drift of the Fine Structure Constant from Optical Frequency Measurements}

\subsubsection{Absolute Frequency Measurements}

Thanks to the optical frequency comb the determination of absolute optical transition frequencies became a simple task, where the attribute "absolute" means that the frequency is measured in hertz, i.e. in terms of the Cs ground state hyperfine splitting. For this a ratio like $f^{(\mathrm{opt})} / f_{\mathrm{Cs}}^{(\mathrm{HFS})}$ is determined. According to Table 1 such a ratio depends on two fundamental constants, $\alpha$ and the Cs nuclear magnetic moment measured in Bohr magneton's $\mu_{\mathrm{Cs}} / \mu_{B}$. One may argue that the latter is not a fundamental quantity, but one has to keep in mind that the nuclear moment is mostly determined by the strong interaction. In that sense it measures the strong interaction in some units. The only difference to the electromagnetic interaction measured by $\alpha$ is that, lacking a precise model for the Cs nucleus we are not sure what those units are. For this reason there are two parameters that need to be determined from an absolute optical frequency measurement and it is impossible to disentangle the contribution from the drift rate of just one absolute optical frequency. At the other hand, the task is solvable if one has more than one absolute frequency measurement at hand under the condition, that the values $L_{\alpha}^{\mathrm{opt}}$ are different for these transitions.

For the general case let's assume that there are $N$ repeated absolute frequency measurements of corresponding transitions $\mathrm{T}_{i}$. For each of the transitions one can derive the relative drift of its absolute frequency $b_{i}(i=1 \ldots N)$ as well as the corresponding uncertainty $\sigma_{i}$ (one standard deviation)

$$
\frac{\partial}{\partial t} \ln \frac{f_{\mathrm{Cs}}^{(\mathrm{HFS})}}{f_{\mathrm{T}_{i}}^{(\mathrm{opt})}}=b_{i} \pm \sigma_{i} .
$$

One can rewrite (15) using the results from Table 2 and (14) 


$$
\begin{aligned}
\frac{\partial}{\partial t} \ln \frac{f_{\mathrm{Cs}}^{(\mathrm{HFS})}}{f_{\mathrm{T}_{i}}^{(\mathrm{opt})}} & =\frac{\partial}{\partial t}\left[\ln \left(\frac{\mu_{\mathrm{Cs}}}{\mu_{B}}\right)+\left(2+L_{\alpha}^{(\mathrm{HFS})}(\mathrm{Cs})-L_{\alpha}^{(\mathrm{opt})}\left(\mathrm{T}_{i}\right)\right) \ln \alpha\right] \\
& =y+A_{i} x
\end{aligned}
$$

where we introduced the definitions $y \equiv \partial \ln \left(\mu_{\mathrm{Cs}} / \mu_{B}\right) / \partial t$ and $x \equiv \partial \ln (\alpha) / \partial t$ respectively. The coefficient $A_{i}$ incorporates sensitivities of the corresponding relativistic corrections $L_{\alpha}$ as well as the $\alpha^{2}$ scaling for hyperfine transitions. Thus, experiments relate $x$ and $y$ to measured values $b_{i}$ with uncertainties $\sigma_{i}$ through:

$$
y=A_{i} x+b_{i} \pm \sigma_{i} .
$$

Let's assume further that the measured data follows a Gaussian distribution $P(x, y)$ :

$$
P(x, y) \propto e^{-\frac{1}{2} R^{2}(x, y)}, \quad \text { where } \quad R^{2}=\sum_{i} \frac{1}{\sigma_{i}^{2}}\left(y-A_{i} x-b_{i}\right)^{2} .
$$

The expectation values for the relative drift rates $x$ and $y$ are determined by the maximum likelihood method corresponding to the minimum of $R^{2}(x, y)$ :

$$
\begin{aligned}
& \frac{\partial R^{2}}{\partial x}=-2 \sum \frac{1}{\sigma_{i}^{2}}\left(y-A_{i} x-b_{i}\right) A_{i}=0 \\
& \frac{\partial R^{2}}{\partial y}=-2 \sum \frac{1}{\sigma_{i}^{2}}\left(y-A_{i} x-b_{i}\right)=0 .
\end{aligned}
$$

With the definitions $B_{1} \equiv \sum 1 / \sigma_{i}^{2}, B_{2} \equiv \sum A_{i}^{2} / \sigma_{i}^{2}, B_{3} \equiv \sum b_{i}^{2} / \sigma_{i}^{2}, B_{4} \equiv \sum A_{i} / \sigma_{i}^{2}$, $B_{5} \equiv \sum b_{i} / \sigma_{i}^{2}, B_{6} \equiv \sum A_{i} b_{i} / \sigma_{i}^{2}$ we can solve system (19) for $x$ and $y$ and obtain expressions for the expectation values:

$$
\langle x\rangle=\frac{B_{4} B_{5}-B_{1} B_{6}}{B_{1} B_{2}-B_{4}^{2}}, \quad\langle y\rangle=\frac{B_{2} B_{5}-B_{4} B_{6}}{B_{1} B_{2}-B_{4}^{2}} .
$$

The standard deviation for $\langle x\rangle$ can be calculated from the integral:

$$
\int_{-\infty}^{+\infty} e^{-\frac{1}{2} R^{2}(x, y)} d y \propto \exp \left[\frac{\left(B_{5}+x B_{4}\right)^{2}-B_{1}\left(B_{3}+x\left(x B_{2}+2 B_{6}\right)\right)}{2 B_{1}}\right] .
$$

Rewriting the exponent

$$
\exp \left[-\frac{(x-\langle x\rangle)^{2}}{2 \sigma_{x}^{2}}+\text { const }_{x}\right]
$$

one gets the standard deviation for $x$

$$
\sigma_{x}=\sqrt{\frac{B_{1}}{B_{1} B_{2}-B_{4}^{2}}}
$$

and, similarly, for $y$

$$
\sigma_{y}=\sqrt{\frac{B_{2}}{B_{1} B_{2}-B_{4}^{2}}}
$$


The evaluation may be represented graphically as on the left hand side of Fig. 2 .

As an example consider the results of the absolute frequency measurements of the $1 S-2 S$ transition in atomic hydrogen (data taken during 2001-2003 at our lab Fischer et al. 2004):

$$
\begin{aligned}
-\frac{\partial}{\partial t} \ln \frac{f_{\mathrm{H}}^{(\mathrm{opt})}}{f_{\mathrm{Cs}}^{(\mathrm{HFS})}} & =\frac{\partial}{\partial t}\left[\ln \left(\frac{\mu_{\mathrm{Cs}}}{\mu_{B}}\right)+(2+0.8) \ln \alpha\right] \\
& =y+2.8 x=(3.2 \pm 6.4) \times 10^{-15} \mathrm{yr}^{-1},
\end{aligned}
$$

the frequency measurement of the electric quadrupole transition $5 \mathrm{~d}^{10} 6 \mathrm{~s}{ }^{2} \mathrm{~S}_{1 / 2}(F=0)-$ $5 \mathrm{~d}^{9} 6 \mathrm{~s}^{2}{ }^{2} \mathrm{D}_{5 / 2}\left(F^{\prime}=2, m_{F}^{\prime}=0\right)$ at $\lambda=282 \mathrm{~nm}$ in a single laser cooled ${ }^{199} \mathrm{Hg}^{+}$ion (data taken during 2000-2006 at NIST (Fortier et al. 2007)):

$$
\begin{aligned}
-\frac{\partial}{\partial t} \ln \frac{f_{\mathrm{Hg}}^{(\mathrm{opt})}}{f_{\mathrm{Cs}}^{(\mathrm{HFS})}} & =\frac{\partial}{\partial t}\left[\ln \left(\frac{\mu_{\mathrm{Cs}}}{\mu_{B}}\right)+(2+0.8+3.2) \ln \alpha\right] \\
& =y+6 x=(-0.37 \pm 0.39) \times 10^{-15} \mathrm{yr}^{-1}
\end{aligned}
$$

and the frequency measurement of the $6 \mathrm{~s}^{2} \mathrm{~S}_{1 / 2}(F=0)-6 \mathrm{~s}^{2} \mathrm{D}_{3 / 2}(F=3)$ electric quadrupole transition at $\lambda=436 \mathrm{~nm}$ of a single trapped and laser cooled ${ }^{171} \mathrm{Yb}^{+}$ion (data taken during 2000-2006 at PTB (Peik et al. 2004, 2006):

$$
\begin{aligned}
-\frac{\partial}{\partial t} \ln \frac{f_{\mathrm{Yb}}^{(\mathrm{opt})}}{f_{\mathrm{Cs}}^{(\mathrm{HFS})}} & =\frac{\partial}{\partial t}\left[\ln \left(\frac{\mu_{\mathrm{Cs}}}{\mu_{B}}\right)+(2+0.8-0.9) \ln \alpha\right] \\
& =y+1.9 x=(0.78 \pm 1.4) \times 10^{-15} \mathrm{yr}^{-1}
\end{aligned}
$$

Using the experimental data from (25), (26), (27) and expressions (20), (23), (24) stringent restrictions for fractional variations of the fundamental constants can be derived (Fortier et al. 2007):

$$
\begin{aligned}
& x=\frac{\partial}{\partial t} \ln \alpha=(-0.31 \pm 0.35) \times 10^{-15} \mathrm{yr}^{-1}, \\
& y=\frac{\partial}{\partial t} \ln \frac{\mu_{\mathrm{Cs}}}{\mu_{B}}=(1.5 \pm 2.0) \times 10^{-15} \mathrm{yr}^{-1} .
\end{aligned}
$$

This result does not use any assumption on correlation of fundamental constants. It is an important advantage of the method opening the possibility to test some extensions of the grand unification theories where the strong, weak and electromagnetic coupling constants are expected to merge for higher energies. The drifts (if existing) of corresponding coupling constants should be correlated; one can even derive a relation of the drift rates of hadron masses and nuclear $g$-factors that are determined by the strong interaction, and the relative drift rate of the fine structure constant: $\Delta m_{p} / m_{p} \approx \Delta g_{\text {nucl }} / g_{\text {nucl }} \approx \pm 35 \Delta \alpha / \alpha$ (Calmet and Fritzsch 2002a, 2002b). Of course, the theory can be tested only if a non zero drift rate is detected as the relation holds even if none of the constants is actually drifting.

\subsubsection{Coupling to Gravity}

Besides setting an upper limit to a variation of $\alpha$, repeated absolute frequency measurements deliver important information about the coupling between gravity and other fundamental interactions. Since 2005 the ${ }^{1} S_{0}-{ }^{3} P_{0}$ clock transition frequency in ${ }^{87} \mathrm{Sr}$ has been measured 
relative to the Cs standard at three laboratories in Paris, Boulder and Tokyo with gradually improving accuracy (Blatt et al. 2008). In these experiments $\mathrm{Sr}$ atoms are placed at the minima of a periodic optical potential (an "optical lattice") tuned to a selected wavelength (Katori et al. 2003) which prevents the clock transition to be shifted by the optical potential. This generates extremely narrow (down to $2 \mathrm{~Hz}$ at $698 \mathrm{~nm}$ ) optical resonances in a large ensemble of atoms. Results agree at a level of $10^{-15}$ so that this type of optical clocks is among the most accurate.

Besides improvement of the null-result $\dot{\alpha} / \alpha=(-3.3 \pm 3.0) \times 10^{-16} \mathrm{yr}^{-1}$, an upper limit for coupling between gravity and other fundamental interactions was set. The Earth moves on an elliptic orbit in a varying solar gravitational potential with a fractional variation of up to $3.3 \times 10^{-10}$. If the coupling between $\alpha$ and the variation of the gravitational potential $\Delta U(t)$ is assumed to be of the form

$$
\frac{\delta \alpha}{\alpha}=k_{\alpha} \frac{\Delta U(t)}{c^{2}},
$$

where $k_{\alpha}$ is the coupling constant, Blatt et al. (2008) have set a restriction of

$$
k_{\alpha}=(2.5 \pm 3.1) \times 10^{-6} .
$$

The same coupling constant has previously been limited with transitions in dysprosium (see also the beginning of Sect. 3) with approximately half the sensitivity (Ferrell et al. 2007).

Of course, the sensitivity of this type of measurement depends on the fractional variation of the gravitational potential which is rather small for the Sun-Earth system. The idea of performing atomic clock frequency comparisons at larger values of $\Delta U / c^{2}$ was considered previously within the SpaceTime satellite mission (Lämmerzahl and Dittus 2002) in which a fly-by maneuver near Jupiter could increase $\Delta U / c^{2}$ to $5 \times 10^{-7}$.

\subsubsection{Direct Comparison of Optical Frequencies}

Improving the accuracy of absolute optical frequency measurements one eventually encounters the limit set by the stability and accuracy of the best Cs clocks (see Fig. 1). The accuracy of the currently best state-of-the art fountain clocks is around $5 \times 10^{-16}$ (Bize et al. 2005). As the frequency combs are not limiting at this level (see e.g. Zimmermann et al. 2004) direct comparison of two optical clocks (Sect. 2.2.3) can provide improved data if both of these clocks are more accurate than the Cs clocks would be.

Indeed at NIST (Boulder, USA) two of these clocks are available that are based on optical clock transition frequencies in $\mathrm{Hg}^{+}$and $\mathrm{Al}^{+}$(Rosenband et al. 2008; Lorini et al. 2008). The frequency ratio measured with the help of a frequency comb has a relative uncertainty of only $5.2 \times 10^{-17}$ which is an order of magnitude smaller than for any absolute frequency measurement. This breakthrough became possible after implementation of new concepts in probing of clock transitions in cold ions, development of narrow-band lasers and progress in optical frequency transfer. Comparison of the highly relativistic system of $\mathrm{Hg}^{+}$(the sensitivity to $\alpha$ variation is $\left.L_{\alpha}^{\mathrm{opt}}\left(\mathrm{Hg}^{+}\right)=-3.2\right)$ with the nearly non-relativistic system of $\mathrm{Al}^{+}$ $\left(L_{\alpha}^{\mathrm{opt}}\left(\mathrm{Al}^{+}\right)=+0.008\right)$ ) over a time interval of only one year allowed to derive a restriction to the variation of $\alpha$ of

$$
\dot{\alpha} / \alpha=(-1.6 \pm 2.3) \times 10^{-17} \mathrm{yr}^{-1} .
$$

This is the lowest limit obtained yet of any type of measurement and is consistent with zero. On top of that the result is model-independent and opens the possibility to draw conclusions about variations of other fundamental constants when combined with other types of experiments. 


\section{Frequency Combs for Astrophysics}

The laser frequency comb turned out to be not only an indispensable element in laboratory optical frequency measurements, but is also useful tool for astronomical observations. It has been demonstrated recently that a frequency comb with a resolvable large mode spacing can be used as an accurate calibration tool for high-resolution spectrographs. This is particular interesting for astrophysical applications (Steinmetz et al. 2008). An accurate frequency axis for spectrometers is required for a number of sensitive fundamental measurements like testing the drift of redshifts of different astrophysical objects (Sandage 1962), the search for extrasolar planets by the reflex Doppler motion (Mayor and Queloz 1995; Marcy and Butler 1996; Lovis et al. 2006) as well as the search for cosmological variations of fundamental constants (Bahcall and Salpeter 1965; Thompson 1975; Webb et al. 1999).

If Doppler shifts on the order of $1 \mathrm{~cm} \mathrm{~s}^{-1}\left(3 \times 10^{-11} c\right)$ could be measured the presumed acceleration of the cosmic expansion could be verified in real time in a largely model independent way, i.e. without assuming the validity of general relativity (Steinmetz et al. 2008). For a typical high-resolution spectrometer used in astrophysics such a resolution corresponds to a physical size of one silicon atom on a CCD substrate. This indicates that only with the statistics of a very large number of calibration lines the sensitivity can be achieved at the condition that the systematics are under control at the same level.

In 2008 a first implementation of the laser frequency comb as a calibration tool for the German Vacuum Tower Telescope (VTT) (Schröter et al. 1985) has been demonstrated (Steinmetz et al. 2008). The very high resolution of the VTT $(0.8 \mathrm{GHz})$ is still too low to resolve individual modes of the erbium fiber laser frequency comb with $\omega_{r}=$ $2 \pi \times 250 \mathrm{MHz}$ used for its calibration. Filtering of the desirable frequency comb modes by an external Fabry-Pérot cavity was suggested as one possible solution (e.g. Li et al. 2008). The Fabry-Pérot cavity used by Steinmetz et al. (2008) has a free spectral range of $m \omega_{r}$ where the integer $m$ can be set between 4 and 60. This cavity interferometrically suppresses all modes generated by the laser except every $m$ th. The resulting well resolved comb is used to illuminate the spectrograph slit.

A CCD image of the fragment of the solar spectrum with atmospheric absorption lines is presented in Fig. 10. The filtered frequency comb radiation was overlaid with the output of the telescope and sent to the VTT spectrometer. The comb modes were stabilized to a Rb atomic clock. One can recognize the resolved filtered comb modes used as frequency markers separated by $15 \mathrm{GHz}$ intervals $(m=60)$ super imposed on the solar spectrum. Even for
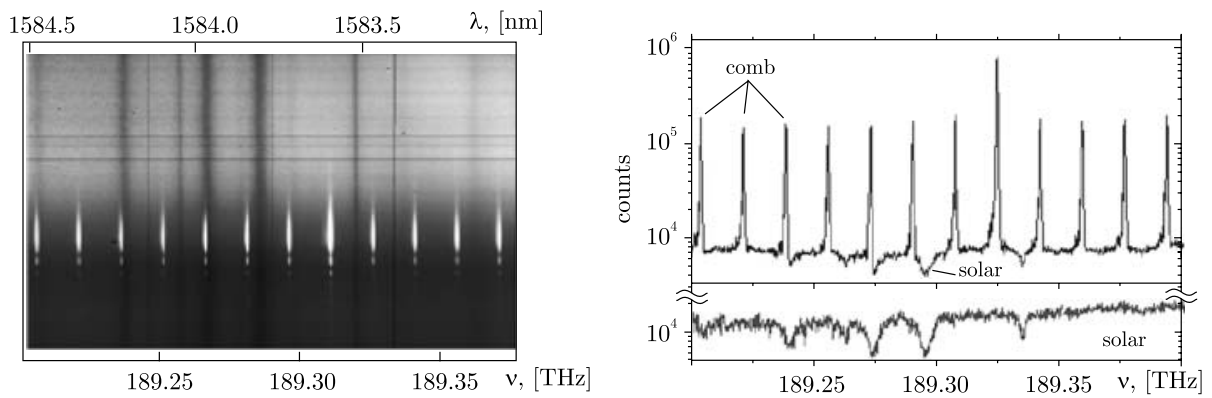

Fig. 10 (Left) - a CCD image of a fragment of the solar photosphere spectrum (dark Fraunhofer lines) overlaid with a frequency comb with $15 \mathrm{GHz}$ mode spacing (bright regular lines). (Right)—horizontal cuts through the CCD image that contain the frequency comb and solar spectrum (top) and the solar spectrum only (bottom) 
Table 3 Model-independent restrictions of the variation of the fine structure constant $\alpha$ from laboratory measurements. Results of Fischer et al. (2004); Peik et al. (2004, 2006), Blatt et al. (2008) were obtained by absolute optical frequency measurements, the result of Rosenband et al. (2008) was obtained by direct comparison of two optical frequencies with the help of a frequency comb, while in Cingöz et al. (2007) a radio-frequency transition between highly-excited nearly degenerative levels has been measured.

\begin{tabular}{|c|c|c|c|c|}
\hline Year & Atomic samples & $\dot{\alpha} / \alpha, \mathrm{yr}^{-1}$ & Method & Ref. \\
\hline 2004 & $\mathrm{H}, \mathrm{Yb}^{+}, \mathrm{Hg}^{+}$ & $(-0.9 \pm 2.9) \times 10^{-15}$ & absolute frequency & Fischer et al. (2004) \\
\hline 2004 & $\mathrm{H}, \mathrm{Yb}^{+}, \mathrm{Hg}^{+}$ & $(-0.3 \pm 2.0) \times 10^{-15}$ & absolute frequency & Peik et al. (2004) \\
\hline 2006 & $\mathrm{Yb}^{+}, \mathrm{Hg}^{+}$ & $(-2.6 \pm 3.9) \times 10^{-16}$ & absolute frequency & Peik et al. (2006) \\
\hline 2007 & Dy & $(-2.7 \pm 2.6) \times 10^{-15}$ & rf transition & Cingöz et al. (2007) \\
\hline 2008 & $\mathrm{Sr}, \mathrm{H}, \mathrm{Yb}^{+}, \mathrm{Hg}^{+}$ & $(-3.3 \pm 3.0) \times 10^{-16}$ & absolute frequency & Blatt et al. (2008) \\
\hline 2008 & $\mathrm{Hg}^{+}, \mathrm{Al}^{+}$ & $(-1.6 \pm 2.3) \times 10^{-17}$ & direct comparison & Rosenband et al. (2008) \\
\hline
\end{tabular}

this very first demonstration a calibration uncertainty of only $9 \mathrm{~m} \mathrm{~s}^{-1}$ (root mean square) in cosmic velocity units was achieved which compares very well with the uncertainties of traditional calibration techniques. By increasing the number of modes (up to $10^{4}$ ) by a spectrally broader frequency comb, it is feasible to reduce the statistical uncertainty to the desirable $1 \mathrm{~cm} \mathrm{~s}^{-1}$ level. The approach also opens possibilities to analyze the systematic uncertainties of the spectrograph and remove their contribution. Implementation of frequency combs for astrophysics allows to observe small variations of spectral lines on a large time scale referenced directly to the SI unit of hertz.

\section{Conclusions}

Table 3 summarizes the results of recent laboratory measurements aiming at the search for a time varying $\alpha$ (see also Fig. 2, right). Combinations with other precision laboratory measurements like comparisons of fountain clocks (see e.g. Marion et al. 2003) or precision molecular spectroscopy (Amy-Klein et al. 2005) deliver important information on the variation of reduced magnetic moments and the electron-to-proton mass ratio. The field is rapidly evolving and we included only a few results most relevant to the topic reviewed.

As seen from the table, repeated frequency measurements in cold atoms, ions and molecules allow to set stringent restrictions on the variation of fundamental constants. At the moment the sensitivity of these methods resides at a level of $\dot{\alpha} / \alpha \sim 10^{-17} \mathrm{yr}^{-1}$ which is the lowest model-independent restriction at the present epoch. A further increase in sensitivity is expected due to improvements of frequency measurements, the increasing observation time interval, and the increase of the number of atomic samples under study. On the other hand, frequency combs open perspectives for improving the accuracy of astrophysical observations and potentially push forward the sensitivity of astrophysical tests for a variation of $\alpha$ billions of years ago.

Acknowledgements J.A. is supported by EU Marie Curie fellowship. N.K. acknowledges support from MPG and RF Presidential grant MD-887.2008.2 and the Russian Science Support Foundation.

Open Access This article is distributed under the terms of the Creative Commons Attribution Noncommercial License which permits any noncommercial use, distribution, and reproduction in any medium, provided the original author(s) and source are credited. 


\section{References}

F. Adler et al., Opt. Express 12, 5872 (2004)

G.P. Agrawal, Nonlinear Fiber Optics (Academic Press, New York, 2001)

J. Alnis, A. Matveev, N. Kolachevsky, Th. Udem, T.W. Hänsch, Phys. Rev. A 77, 1 (2008)

A. Amy-Klein et al., Opt. Lett. 30, 3320 (2005)

J.N. Bahcall, E.E. Salpeter, Astrophys. J. 142, 1677 (1965)

F. Biraben et al., in The Hydrogen Atom. Precision Physics of Simple Atomic Systems, ed. by S.G. Karshenboim, F.S. Pavone, G.F. Bassani, M. Inguscio, T.W. Hänsch (Springer, Berlin, 2001), p. 18

S. Bize et al., Phys. Rev. Lett. 90, 150802 (2003)

S. Bize et al., J. Phys. B: At. Mol. Opt. Phys. 38, S449-68 (2005)

S. Blatt et al., Phys. Rev. Lett. 100, 140801 (2008)

X. Calmet, H. Fritzsch, Eur. Phys. J. C 24, 639 (2002b)

X. Calmet, H. Fritzsch, Phys. Lett. B 540, 173 (2002a)

H.B.G. Casimir, On the Interaction Between Atomic Nuclei and Electrons (Freeman, San Francisco, 1963), p. 54

H. Chand, R. Srianand, P. Petitjean, B. Aracil, Astron. Astrophys. 417, 853 (2004)

A. Cingöz et al., Phys. Rev. Lett. 98, 040801 (2007)

T. Damour, F. Piazza, G. Veneziano, Phys. Rev. Lett. 89, 081601 (2002)

S.A. Diddams, L. Hollberg, L.S. Ma, L. Robertsson, Opt. Lett. 27, 58 (2002)

S.A. Diddams et al., Phys. Rev. Lett. 84, 5102 (2000)

P.A.M. Dirac, Nature (London) 139, 323 (1937)

R.W.P. Drever et al., Appl. Phys. 31, 97 (1983)

V.A. Dzuba, V.V. Flambaum, Phys. Rev. A 61, $034502(2000)$

V.A. Dzuba, V.V. Flambaum, J.K. Webb, Phys. Rev. A 59, 230 (1999a)

V.A. Dzuba, V.V. Flambaum, J.K. Webb, Phys. Rev. Lett. 82, 888 (1999b)

J.N. Eckstein, A.I. Ferguson, T.W. Hänsch, Phys. Rev. Lett. 40, 847 (1978)

M.I. Eides, H. Grotch, V.A. Shelyuto, Phys. Rep. 342, 63 (2001)

K.M. Evenson, J.S. Wells, F.R. Petersen, B.L. Danielson, G.W. Day, Appl. Phys. Lett. 22, 192 (1973)

S.J. Ferrell et al., Phys. Rev. A 76, 062104 (2007)

M. Fischer et al., Phys. Rev. Lett. 92, 230802 (2004)

V.V. Flambaum, Int. J. Mod. Phys. A 22, 4937 (2007)

V.V. Flambaum, E.V. Shuryak, AIP Conf. Proc. 995, 1 (2008)

T.M. Fortier et al., Phys. Rev. Lett. 98, 070801 (2007)

R.W. Fox, Proc. SPIE 7099, 70991R (2008)

H.A. Haus, E.P. İppen, Opt. Lett. 26, 1654 (2001)

C. Henry, IEEE J. Quantum Electron. 18, 259 (1982)

L.O. Hocker, A. Javan, D.R. Rao, L. Frenkel, T. Sullivan, Appl. Phys. Lett. 10, 147 (1967)

R. Holzwarth et al., Phys. Rev. Lett. 85, 2264 (2000)

R. Holzwarth et al., Laser Phys. 11, 1100 (2001)

A. Huber, Th. Udem, B. Gross, J. Reichert, M. Kourogi, K. Pachucki, M. Weitz, T.W. Hänsch, Phys. Rev. Lett. 80, 468 (1998)

S.G. Karshenboim, V.G. Ivanov, Euro. Phys. J. D 19, 13 (2002b)

S.G. Karshenboim, V.G. Ivanov, Phys. Lett. B 524, 259 (2002a)

H. Katori, M. Takamoto, V.G. Pal'chikov, V.D. Ovsiannikov, Phys. Rev. Lett. 91, 173005 (2003)

J.C. Knight et al., Opt. Lett. 21, 1547 (1996)

N. Kolachevsky, M. Fischer, S.G. Karshenboim, T.W. Hänsch, Phys. Rev. Lett. 92, 033003 (2004a)

N. Kolachevsky, P. Fendel, S.G. Karshenboim, T.W. Hänsch, Phys. Rev. A 70, 063503 (2004b)

P. Kubina et al., Opt. Express 13, 909 (2005)

C. Lämmerzahl, H. Dittus, Ann. Phys. (Leipzig) 11, 95 (2002)

C.-H. Li et al., Nature 452, 610 (2008)

L. Lorini et al., Eur. Phys. J. Special Topics 163, 19 (2008)

C. Lovis et al., Nature 441, 305 (2006)

A.D. Ludlow et al., Opt. Lett. 32, 641 (2007)

L.S. Ma et al., Science 303(5665), 1843 (2004)

G.W. Marcy, R.P. Butler, Astrophys. J. 464, L147 (1996)

H. Marion et al., Phys. Rev. Lett. 90, 150801 (2003)

M. Mayor, D. Queloz, Nature 378, 355 (1995)

R. Minkowski, O.C. Wilson, Astrophys. J. 123, 373 (1956)

M.T. Murphy, J.K. Webb, V.V. Flambaum, Mon. Not. R. Astron. Soc. 384, 1053 (2008) 
E. Narevicius, A. Libson, C.G. Parthey, I. Chavez, J. Narevicius, U. Even, M.G. Raizen, Phys. Rev. Lett. 100, 093003 (2008)

T. Nazarova, F. Riehle, U. Sterr, Appl. Phys. B 83, 531 (2006)

L.E. Nelson et al., Appl. Phys. B 65, 277 (1997)

M. Niering et al., Phys. Rev. Lett. 84, 5496 (2000)

M. Notcutt, L.-S. Ma, J. Ye, J.L. Hall, Opt. Lett. 30, 1815 (2005)

E. Peik et al., Phys. Rev. Lett. 93, 170801 (2004)

E. Peik et al., in Proceedings of the 11th Marcel Grossmann Meeting, Berlin (2006). arXiv:physics/0611088

K. Petermann, IEEE J. Quantum Electron. 15, 566 (1979)

R. Quast, D. Reimers, S.A. Levshakov, Astron. Astrophys. 415, 27 (2004)

J. Reichert, R. Holzwarth, Th. Udem, T.W. Hänsch, Opt. Commun. 172, 59 (1999)

J. Reichert et al., Phys. Rev. Lett. 84, 3232 (2000)

T. Rosenband et al., Science 319, 1808 (2008)

A. Sandage, Astrophys. J. 136, 319 (1962)

G. Santarelli et al., Phys. Rev. Lett. 82, 4619 (1999)

M.P. Savedoff, Nature 178, 689 (1956)

A.L. Schawlow, C.H. Townes, Phys. Rev. 112, 1940 (1958)

H. Schnatz, B. Lipphardt, J. Helmcke, F. Riehle, G. Zinner, Phys. Rev. Lett. 76, 18 (1996)

E.H. Schröter, D. Soltau, E. Wiehr, Vistas Astron. 28, 519 (1985)

T. Steinmetz et al., Science 321, 1335 (2008)

H. Stoehr, F. Mensing, J. Helmcke, U. Sterr, Opt. Lett. 31, 736 (2006)

T.R. Taylor, G. Veneziano, Phys. Lett. B 213, 450 (1988)

R.I. Thompson, Astron. Lett. 16, 3 (1975)

Th. Udem, R. Holzwarth, T.W. Hänsch, Nature 416, 233 (2002)

Th. Udem, J. Reichert, R. Holzwarth, T.W. Hänsch, Opt. Lett. 24, 881 (1999)

N. Vanhaecke, U. Meier, M. Andrist, B.H. Meier, F. Merkt, Phys. Rev. A 75, 031402(R) (2007)

D.A. Varshalovich, A.V. Ivanchik, A.V. Orlov, A.Y. Potekhin, P. Petitjean, Current status of the problem of cosmological variability of fundamental physical constants, in Lecture Notes in Physics: Precision Physics of Simple Atomic Systems, ed. by S.G. Karshenboim, V.B. Smirnov (Springer, Berlin, 2003), pp. 199

J.K. Webb, V.V. Flambaum, C.W. Churchill, M.J. Drinkwater, J.D. Barrow, Phys. Rev. Lett. 82, 884 (1999)

J.K. Webb et al., Phys. Rev. Lett. 87, 091301 (2001)

M. Weitz, A. Huber, F. Schmidt-Kaler, D. Leibfried, T.W. Hänsch, Phys. Rev. Lett. 72, 328 (1994)

B.C. Young, F.C. Cruz, W.M. Itano, J.C. Bergquist, Phys. Rev. A 82, 3799 (1999)

M. Zimmermann, C. Gohle, R. Holzwarth, Th. Udem, T.W. Hänsch, Opt. Lett. 29, 310 (2004) 